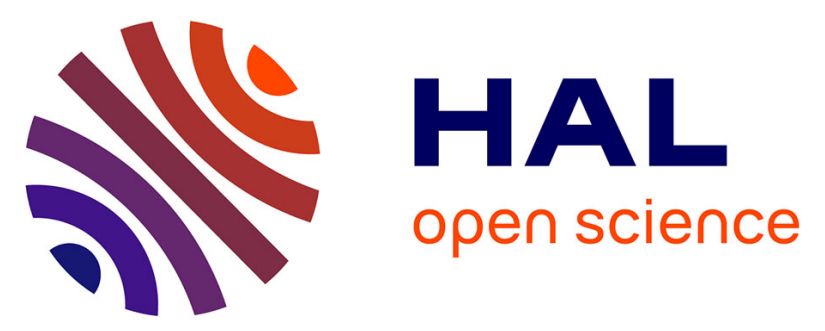

\title{
Electrode Selection for Noninvasive Fetal Electrocardiogram Extraction using Mutual Information Criteria
}

Reza Sameni, Frédéric Vrins, F. Parmentier, Christophe Herail, Vincent Vigneron, Michel Verleysen, Christian Jutten, Mohammad B. Shamsollahi

\section{To cite this version:}

Reza Sameni, Frédéric Vrins, F. Parmentier, Christophe Herail, Vincent Vigneron, et al.. Electrode Selection for Noninvasive Fetal Electrocardiogram Extraction using Mutual Information Criteria. 26th International Workshop on Bayesian Inference and Maximum Entropy Methods in Science and Engineering (MaxEnt 2006), Jul 2006, Paris, France. pp.97-104, 10.1063/1.2423265 . hal-00966350

\section{HAL Id: hal-00966350 \\ https://hal.science/hal-00966350}

Submitted on 26 Mar 2014

HAL is a multi-disciplinary open access archive for the deposit and dissemination of scientific research documents, whether they are published or not. The documents may come from teaching and research institutions in France or abroad, or from public or private research centers.
L'archive ouverte pluridisciplinaire $\mathbf{H A L}$, est destinée au dépôt et à la diffusion de documents scientifiques de niveau recherche, publiés ou non, émanant des établissements d'enseignement et de recherche français ou étrangers, des laboratoires publics ou privés. 


\title{
Electrode Selection for Noninvasive Fetal Electrocardiogram Extraction using Mutual Information Criteria
}

\author{
R. Sameni ${ }^{* \dagger}$, F. Vrins ${ }^{* *}$, F. Parmentier**, C. Hérail ${ }^{*}$, V. Vigneron ${ }^{*}$, \\ M. Verleysen ${ }^{* *}$, C. Jutten* and M. B. Shamsollahi \\ *Laboratoire des Images et des Signaux (LIS) - CNRS UMR 5083, INPG, UJF, Grenoble, France \\ ${ }^{\dagger}$ Biomedical Signal and Image Processing Laboratory (BiSIPL), School of Electrical Engineering, \\ Sharif University of Technology, Tehran, Iran \\ ** Machine Learning Group (MLG), Microelectronics Laboratory, Université Catholique de \\ Louvain (UCL), Louvain-La-Neuve, Belgium \\ ${ }^{\ddagger}$ Laboratoire Informatique Biologie Intégrative et Systèmes Complexes (IBISC) - CNRS FRE 2873, Evry, \\ France
}

\begin{abstract}
Blind source separation (BSS) techniques have revealed to be promising approaches for the noninvasive extraction of fetal cardiac signals from maternal abdominal recordings. From previous studies, it is now believed that a carefully selected array of electrodes well-placed over the abdomen of a pregnant woman contains the required 'information' for BSS, to extract the complete fetal components. Based on this idea, previous works have involved array recording systems and sensor selection strategies based on the Mutual Information (MI) criterion. In this paper the previous works have been extended, by considering the 3-dimensional aspects of the cardiac electrical activity. The proposed method has been tested on simulated and real maternal abdominal recordings. The results show that the new sensor selection strategy together with the MI criterion, can be effectively used to select the channels containing the most 'information' concerning the fetal ECG components from an array of 72 recordings. The method is hence believed to be useful for the selection of the most informative channels in online applications, considering the different fetal positions and movements.
\end{abstract}

Keywords: Fetal Electrocardiogram, ICA, Mutual Information

PACS: $87.80 . \mathrm{Tq}$

\section{INTRODUCTION}

In recent years, blind source separation (BSS) techniques have found special interests in the biomedical signal processing community. Specifically, BSS has been very effective for the noninvasive extraction of fetal cardiac signals from maternal abdominal recordings [1], where conventional filtering schemes do not have satisfactory performances. From the related state-of-the-art, it is believed that the complete shape of the fetal ECG should be extractable from a sufficient number of recordings captured by electrodes suitably positioned over the abdomen of pregnant woman. Based on this idea, in previous research multi-channel recording systems containing up to 72 electrodes have been developed, which can be placed as a belt of electrodes over the abdomen and the back of pregnant woman [2]. However, the fetal cardiac signals are not directly extractable from such recordings, for two reasons. First, many of the recording channels are contaminated with high-power noises such as the maternal ECG and contain little information about the fetal ECG. Second, the processing of all the different combinations of these 
electrodes $(72 \times 71 / 2$ electrode pairs $)$, can be very time-consuming and inefficient, since a much smaller subset of the electrodes (which can also vary with time depending on the location of the fetus, shape of the abdomen, or stage of pregnancy), may be sufficient to extract the required 'information'. Based on these facts, an electrode selection strategy was recently proposed to reject the channels which correspond to the maternal ECG, by minimizing the mutual information (MI) between the different electrodes and a reference channel reflecting the 'pure' maternal ECG [3]. The method was proposed as a preprocessing for applying ICA to the multi-channel recordings.

On the other hand according to the dipole theory of the heart, it is known that the electrodes placed on the body surface receive projections of the cardiac dipole vectors, depending on their relative positions with respect to the maternal and fetal hearts. This suggests that the 'pure' maternal and fetal signals are not unique for all electrodes. Consequently the recordings obtained from each of the abdominal electrodes should be compared with their own corresponding reference, rather than a single reference that is shared by all the sensors.

In this work, the problem of electrode selection has been revisited, by considering the 3-dimensional shapes of the cardiac dipole. For this, the reference channels have been customized for each of the recording channels and the MI of each channel has been calculated with respect to its corresponding reference. Moreover, the channel selection strategy has been modified by preserving the channels having the lowest MI with the associated maternal ECGs and, at the same time, the highest MI with the fetal ECG to avoid selecting highly noisy signals. The effectiveness of the proposed method has been verified by extracting the fetal components from the selected electrodes by using ICA.

\section{BACKGROUND}

\section{The Vectorcardiogram vs. the Electrocardiogram}

The electrical activity of the cardiac muscle and its relationship with the body surface potentials, namely the Electrocardiogram (ECG), has been modeled with different approaches ranging from single dipole models (SDM), to activation maps [4]. Among these methods, the simplest and yet the most popular is the SDM, which is believed to explain $80 \%-90 \%$ of the representation power of the body surface potentials [5]. The ECG and the vectorcardiogram (VCG) are also based on this model. According to the SDM, the cardiac electrical activity may be represented by a time-varying rotating vector, with its origin located at the center of the heart and its end sweeping a quasi-periodic region in the space. The dipole model is a mean of modeling the heart source, and in order to analyze the electrical recordings on the body surface, an additional model is required for the propagation of the heart potentials in the body volume conductor. By assuming this volume conductor as a passive resistive medium which only attenuates the source field (no delays, no reverberations, etc), any ECG signal would simply be a linear projection of the cardiac dipole onto the direction of the recording electrode axes.

A 3-D vector representation of the ECG, namely the VCG, is also possible by using three of such ECG signals. Basically any three linearly independent set of ECG electrodes can be used to construct the VCG. However in order to achieve an orthonormal 
representation which resembles the dipole vector, a set of three orthogonal electrodes which best match to the three body axes are selected. The Frank lead system, or the corrected Frank lead system which has better orthogonality and normalization, are conventional means of recording the VCG [6]. In a recent work [7], a realistic model of the adult ECG and maternal abdominal recordings has been developed based on the SDM. The model consists of 3-dimensional dynamic models for the maternal and fetal cardiac dipoles and the maternal body volume conductor. We have used this model to verify the effectiveness of the MI criterion for localizing the best sensors which contain the most information about the fetal heart with the least maternal ECG contaminants.

\section{Application of Independent Components Analysis and Mutual Information for ECG Signals}

Mathematically Independent Component Analysis (ICA) always gives a solution for the most independent sources contributing to a linear mixture of signals. However when applying ICA to multivariate ECG recordings, the interpretation of the extracted sources and the extraction of meaningful clinical measures from these components need closer study of the ECG and the VCG. In some previous studies, it has been shown that by using the dipole model of the heart, the body surface potentials can be linearly related to the cardiac dipoles $[1,5,8]$. In [8], it is further shown that with appropriate baseline wander removal, the columns of the mixing matrix extracted by ICA correspond to the most representative axes of the VCG loop scatter, which are closely related to the directions of the main planes in the VCG space.

There are several means of measuring the independence between random variables [9], however the Mutual Information (MI) has interesting properties that are specifically suitable for the problem of interest [10]. Considering two random vectors (or scalars) $X$ and $Y$, with the joint probability density function $p_{X, Y}(x, y)$, and the marginal densities $p_{X}(x)$ and $p_{Y}(y)$, the MI is defined as follows:

$$
I(X, Y)=\iint p_{X, Y}(x, y) \log \frac{p_{X, Y}(x, y)}{p_{X}(x) p_{Y}(y)} d x d y .
$$

One of the interesting properties of MI, is the independence with respect to reparameterizations [10]. This means that if $X^{\prime}=F(X)$ and $Y^{\prime}=G(Y)$ are invertible transformations, we have:

$$
I\left(X^{\prime}, Y^{\prime}\right)=I(X, Y)
$$

These equations hold for both scalar and vector variables of arbitrary dimensions [10].

According to the SDM and by assuming a linear propagation medium, up to $80 \%-$ $90 \%$ of the representation power of the body surface potentials can be achieved through a linear combination of these signals. This implies that with a good approximation, the VCG representations constructed from any set of 3 linearly independent reference ECG leads can be linearly mapped to the standard orthonormal VCG representation $V C G_{0}(t)$, as follows:

$$
V C G(t) \approx A \cdot V C G_{0}(t),
$$


where the approximation results from assumptions of SDM that are not exactly fulfilled in practice. In this model the $3 \times 3$ transformation matrix $A$ depends on the location of the electrodes and the attenuation of the body volume conductor. The well-known Dower transformation and its inverse which are used to map the standard ECG leads and the Frank leads, are the evident results of this approximation [11]. Now by using (1) and (2), if we calculate the MI between any noisy recording $x(t)$ and any set of reference VCGs (neither necessarily orthogonal, nor orthonormal), we find the following result:

$$
I(x(t), V C G(t)) \approx I\left(x(t), A \cdot V C G_{0}(t)\right)=I\left(x(t), V C G_{0}(t)\right),
$$

where the approximation is again due the approximation in (3). Moreover according to (2) the transformation between $V C G(t)$ and $V C G_{0}(t)$ only needs to be invertible and not necessarily linear. The interesting interpretation of this equation is that as long as a unique and invertible transformation (either linear or nonlinear), exists between two sets of VCG recordings, its MI with any of the recording channels is robust to the changes and movements of the reference VCG channels. This point is practically important, as the reference electrodes can either move or have slight location differences in different recordings.

\section{ELECTRODE SELECTION STRATEGIES}

As explained before, there are totally 71 channels of real recordings available. In practice, it happens that some of the electrodes are detached from the maternal body, making there corresponding channel totally noisy. These electrodes should be eliminated before the main processing, leaving $N \leqslant 71$ channels for the processing.

The electrode selection strategy originally suggested in [3], uses a unique reference signal for the rejection of the channels which contain the most mutual information with the maternal ECG. However according to the 3D representation of the VCG presented in previous sections, it is now clear that the different electrodes are recording the projection of the cardiac potentials from different angles. Hence, although they are all time-synchronized, the maternal ECG contribution in the recordings may have totally different shapes depending on the associated sensor's location. This means that a unique maternal reference template is not sufficient for all channels. Ideally, we would like to have an appropriate reference channel for each of the electrodes. Unfortunately the idea of using a specific reference for each electrode can only be achieved on simulated data, and it is not applicable to real ECG recordings. However, a suitable compromise is to use a set of 3 reference recordings such as the 3 signals captured from the standard Frank lead electrodes and compare each of the channels with its corresponding reference.

For real recordings, in order to select the channels with the least interference from the maternal components, two experiments have been carried out. In the first experiment we select the set of reference electrodes $\mathscr{R}=\left\{r_{1}(t), r_{2}(t), r_{3}(t)\right\}$, being three typical signals obtained via the Frank lead sensors from a pregnant woman. For the case where the Frank lead recordings are not valid, synthetic Frank lead references can be generated by passing a train of pulses which are time-synchronized with the R-peaks of the mother, through systems with impulse responses similar to the typical signals of the Frank lead electrodes. The output of this system can be used as the three reference channels for the 
maternal ECG. Using these reference signals, each of the $N$ channels are classified to one of the three classes labelled 1,2 , and 3 , as follows:

$$
\forall x \in \mathscr{X}, \mathscr{C}(x) \doteq \underset{j \in\{1,2,3\}}{\operatorname{argmax}}\left|\operatorname{corr}\left(x, r_{j}\right)\right|, r_{j} \in \mathscr{R} .
$$

Accordingly, each channel is assigned to the class with which it has the largest correlation with its reference signal, and for further calculations the MI is calculated between each channel and its corresponding class reference. This pre-classification is used in the following iterative channel selection.

Assuming that $\mathscr{X}^{\prime}=\left\{x_{1}^{\prime}, \ldots, x_{k-1}^{\prime}\right\}$ is the set of $k-1$ already selected signals, the $k$-th signal $x_{k}^{\prime}$ is selected as follows:

$$
x_{k}^{\prime} \doteq \underset{x_{j} \in \mathscr{X} \backslash \mathscr{X}^{\prime}}{\operatorname{argmin}}\left\{I\left(x_{j}, r_{\mathscr{C}(x)}\right)+\sum_{i=1}^{k-1} I\left(x_{i}^{\prime}, x_{j}\right)\right\}, x_{i}^{\prime} \in \mathscr{X}^{\prime}, r_{\mathscr{C}(x)} \in \mathscr{R} \quad \text { (Maternal rule 1). }
$$

The intuition behind the first term in this equation is to find the most independent channels (in terms of MI), which contain the least information about the maternal components, while the second term assures that the selected channels are not redundant and contain information which was not provided by the previously selected channels.

In a second experiment, we artificially extend the number of references from $n(\mathscr{R})=3$ to $n(\mathscr{R})=N$ as follows. According to the SDM explained in previous sections, if only the maternal heart was active, the signals recorded from the maternal body could be approximated by:

$$
\mathbf{x}(t) \approx H \times V C G(t),
$$

where $\mathbf{x}(t) \in \mathbb{R}^{N}$ is the array of recorded signals, $V C G(t) \doteq\left[r_{1}(t), r_{2}(t), r_{3}(t)\right]^{T}$ is the $3 \mathrm{D}$ maternal VCG that can be obtained via the Frank orthonormal representation, and $H \in \mathbb{R}^{N \times 3}$ is the transfer matrix depending on the propagation medium and sensor locations, which may be found by solving the following least squares problem:

$$
\hat{H} \doteq \underset{H}{\operatorname{argmin}}\|\mathbf{x}(t)-H \times V C G(t)\| .
$$

Note that if we consider $\hat{h}_{i}$ as the $i^{t h}$ row of $\hat{H}, \hat{h}_{i} \times V C G(t)$ is the local maternal ECG reference for the $i^{t h}$ recorded signal $x_{i}(t)$. This result leads us towards the second sensor selection strategy. Again, assuming that $\mathscr{X}^{\prime}=\left\{x_{1}^{\prime}, \ldots, x_{k-1}^{\prime}\right\}$ is the set of $k-1$ selected signals, the $k$-th signal $x_{k}^{\prime}$ is selected as follows:

$$
x_{k}^{\prime} \doteq \underset{x_{j} \in \mathscr{X} \backslash \mathscr{X}^{\prime}}{\operatorname{argmin}}\left\{I\left(x_{j}, \hat{h}_{i} \times V C G(t)\right)+\sum_{i=1}^{k-1} I\left(x_{i}^{\prime}, x_{j}\right)\right\}, \forall i, x_{i}^{\prime} \in \mathscr{X}^{\prime} \quad \text { (Maternal rule 2). }
$$

Using any of the maternal rules (1) or (2), the channels containing the least MI with the maternal signals are achieved. However this is not enough to ensure that a channel contains fetal components, since highly noisy signals which neither correspond to the maternal nor the fetal components can also be selected by such criteria. In order to solve this problem, we need some additional information concerning the fetal components. 
For the moment, lets suppose that we had one or more fetal references defined by the set $\mathscr{R}^{\prime}$, similar to the maternal references considered above. In this case, the signal which contains the most information about the fetal components could be found as follows:

$$
x^{\prime \prime} \doteq \underset{x_{j}^{\prime} \in \mathscr{X}^{\prime}}{\operatorname{argmax}}\left\{I\left(x_{j}^{\prime}, r_{\mathscr{C}\left(x_{j}^{\prime}\right)}\right)\right\}, \quad r_{\mathscr{C}\left(x_{j}^{\prime}\right)}^{\prime} \in \mathscr{R}^{\prime} \quad \text { (Fetal rule 1). }
$$

This rule may be applied several times in order to find the best $M<n\left(\mathscr{X}^{\prime}\right)$ channels containing the most information about the fetus, by each time removing the selected channel from $\mathscr{X}^{\prime}$ and applying the rule to its remaining members. Note that in the fetal rule, we no longer considered the minimization of the mutual information with the previously selected channels, since the fetal components are very weak and even minor information concerning the fetal components should not be lost.

Now the remaining problem is how to find a set of fetal reference potentials. As mentioned before, for simulated recordings we have the fetal and maternal references for each channel; however for real recordings no a priori reference is available for the fetus. In order to find a fetal reference we can apply ICA to the total $N$-channel database or to the channels selected by the maternal rules. This usually provides at least one channel which corresponds to the fetal components and may be used as a single fetal reference. Moreover the R-peaks of the fetal components may also be detected from this single component and used to make a synchronous averaging of the different channels. In fact due to the quasi-periodic shape of the ECG, when we average the different channels synchronous with the fetal R-peaks, the SNR of the fetal components are improved; leading to an average ECG waveform which can be used as the reference for each channel. Next, as mentioned before, we can use these average waveforms together with the R-peak pulses to achieve synthetic references for each channel ${ }^{1}$.

\section{RESULTS}

The recording system consists of 72 electrodes placed in a grid of 8 columns and 9 rows throughout the maternal abdomen and back. The signals recorded from each of the 2 neighboring electrode pairs are amplified by a differential amplifier and sampled at $1 \mathrm{kHz}$ with a resolution of 12-bits. Accordingly 71 differential channels are achieved, which are transferred to a computer for further processing. Note that, from this electrode configuration, it is possible to reconstruct the potential difference between any pair of the original recording electrodes, through a linear combination of the recorded signals, meaning that a total combination of $72 \times 71 / 2$ differential pair of recordings is possible.

Some of the electrode selection strategies developed in the previous section have been tested on real recordings. The studied database consisted of 71 recordings. Due to the detachments of some electrodes from the skin of the pregnant woman, 13 of these channels were totally noisy channels which were detected and removed through the preprocessing. From the remaining set $\mathscr{X}$ of 58 channels, the maternal R-peaks

\footnotetext{
1 The idea of synchronous averaging may also be used as an alternative means of achieving the maternal references for each channel. However we have not considered this approach in this paper.
} 


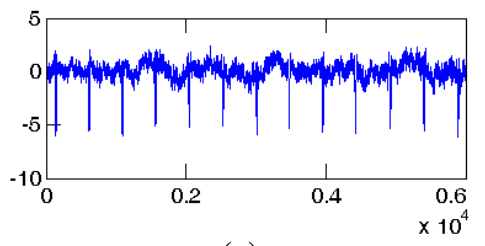

(a)

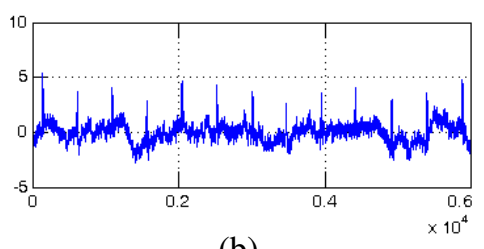

(b)

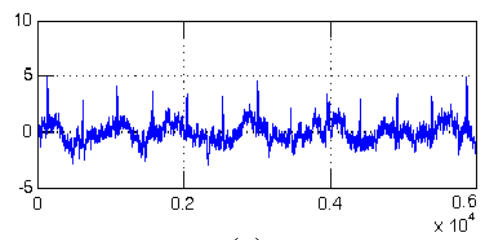

(c)

FIGURE 1. Typical segment of fetal ECG components extracted by ICA from various sets of sensors using the ICA inputs as (a) $\mathscr{X}$; the 58 signals with relevant information, (b) the 20 signals selected using maternal rule (1) from $\mathscr{X}$, (c) ICA inputs were the 10 signals selected by applying fetal rule (1) from the 20 ones obtained through maternal rule (1).

were detected and the three Frank reference signals were generated by using the Rwave train of pulses. Using these adapted references, these channels were classified into one of the three classes based on equation (5). In this stage, 47 of the channels were classified to the first class, 6 to the second, and 5 to the third. Next the 20 ones having the least interference from the maternal ECGs were selected by using maternal rule (1). From these 20 channels, 6 were chosen with fetal rule (1); for this stage, the fetal component obtained by running FastICA on the 58 recordings set was chosen as a unique fetal reference ${ }^{2}$. This 'full-set' ICA has only to be applied once, to build the reference, which can then be used exactly as the Frank leads. Note that if one selects 10 signals from $\mathscr{X}$ using maternal rule (1), JADE does not retrieve any fetal component from the selected recordings; without the fetal rule (1), 20 signals have to be selected by maternal rule (1) in order that JADE gives a fetal component. By contrast, our results indicate that selecting 10 signals using maternal rule (2) from $\mathscr{X}$ is sufficient: a fetal rule is not needed here. Indeed, both methods maternal rule (1) + fetal rule (1), and the maternal rule (2) alone, give globally similar signals. Specifically for $K=10,7$ sensors are common to both sets. Typical fetal components extracted by the different methods can be seen in Fig. 1. We can see in this figure that the SNR is slightly better in the signal extracted from the total 58 channels; but the fetal components have still been extracted by using a selected subset of 10 channels.

\section{DISCUSSIONS AND CONCLUSIONS}

In this paper, the extraction of the fetal ECG from a large array of sensors is investigated in the ICA framework. In order to address this problem in real-time, a selection of few sensors from the array is considered. In order to perform an ICA method on a subset of say $k \ll N$ recordings, an ad-hoc selection strategy has to be developed, since applying ICA on a randomly selected subset of $k$ recordings does not give a fetal component, except when $k$ is rather high (close to $N$ ). This paper extends previous works on sensor selection strategies. The presented approaches use multidimensional references inspired

\footnotetext{
${ }^{2}$ It should be noted that in this stage, due to the high dimension of the dataset, FastICA was preferred to JADE.
} 
by a detailed analysis of the cardiac signals and their dimensionality. The proposed strategy is proved on real-world data. Contrary to a random selection technique, our method gives a small subset which contains fetal information. Indeed, in spite of a strong dimensionality reduction of the ICA input space, the fetal component has been retrieved. The method is hence believed to be useful for the selection of the most informative channels in online applications, considering the different fetal positions and movements.

\section{REFERENCES}

1. L. D. Lathauwer, B. D. Moor, and J. Vandewalle, IEEE Trans. Biomed. Eng. 47, 567-572 (2000).

2. F. Vrins, V. Vigneron, C. Jutten, and M. Verleysen, "Abdominal Electrodes Analysis by Statistical Processing for Fetal Electrocardiogram Extraction," in Proc. 2nd IASTED Int. Conf. on Biomedical Engineering (BioMed 2004), Innsbruck, Austria, 2004, pp. 244-249.

3. F. Vrins, C. Jutten, and M. Verleysen, "Sensor Array and Electrode Selection for Non-Invasive Fetal Electrocardiogram Extraction by Independent Component Analysis," in Lecture Notes in Computer Science (LNCS 3195), Independent Component Analysis and Blind Signal Separation, Proceedings of ICA'04, the 5th Int. Conf. on Independent Component Analysis, Granada, Spain, 2004, pp. 10171024.

4. O. Dössel, Int. J. Bioelectromagnetism 2 (2000), URL \{ http: / / www . i jbem . org/volume2/ number2/doessel/paper.htm .

5. A. van Oosterom, 100 years Einthoven pp. 7-15 (2002).

6. J. A. Malmivuo, and R. Plonsey, editors, Bioelectromagnetism, Principles and Applications of Bioelectric and Biomagnetic Fields, Oxford University Press, 1995, URL http://butler.cc. tut.fi/ malmivuo/bem/bembook.

7. R. Sameni, G. D. Clifford, C. Jutten, and M. B. Shamsollahi, Multi-Channel ECG and Noise Modeling: Application to Maternal and Fetal ECG Signals (2006), submitted to the EURASIP Journal of Applied Signal Processing.

8. R. Sameni, , C. Jutten, and M. B. Shamsollahi, What ICA Provides for ECG Processing: Application to Noninvasive Fetal ECG Extraction (2006), accepted for the 6th IEEE International Symposium on Signal Processing and Information Technology, Aug. 27-30, 2006, Vancouver, Canada.

9. A. Hyvärinen, J. Karhunen, and E. Oja, Independent Component Analysis, Wiley-Interscience, 2001.

10. A. Kraskov, H. Stoegbauer, and P. Grassberger, Physical Review 69, 066138 (2004).

11. L. Edenbrandt, and O. Pahlm, J. Electrocardiol. 21, 361 (1988). 